\title{
Three-tier Design Model of Web Application Based on ASP.net Technology
}

\author{
Xiaoyong Ding \\ Shangrao Preschool Education College, 334000, China
}

Keywords: ASP.Net Technology, Web, Application, Three-Tier Design Model.

\begin{abstract}
Web the application of ASP.net technology in the design of applications, is to use a hierarchical approach in the design of programs, layers and layers are independent, but also linked to each other, the structure of the program is tight, and looks clear and brief, which is very beneficial to the operation and maintenance of the program. In the actual application development work, we should clearly divide the three-tier structure from the specific situation. This paper aims at the research of three-layer design model of Web application based on ASP.net technology.
\end{abstract}

\section{Introduction}

The use of ASP.net technology on Microsoft application platform is a supplement to traditional web, which has strong vitality.and this technology plays a role in microsoft application platform, has good compatibility, can use functions for all functions of. net Framewor, can compile dynamically, and also has type security functions. Compared with traditional technology, ASP.net technology still has an important advantage, which is to support the programming of strong type language, which is helpful for the development of object-oriented programming. There are also defects in the application of ASP.net technology, that is, in fact, using scripting language to write control logic, combined with HTML tags, which will lead to high difficulty in page development.(Figure 1: ASP.net technical model)
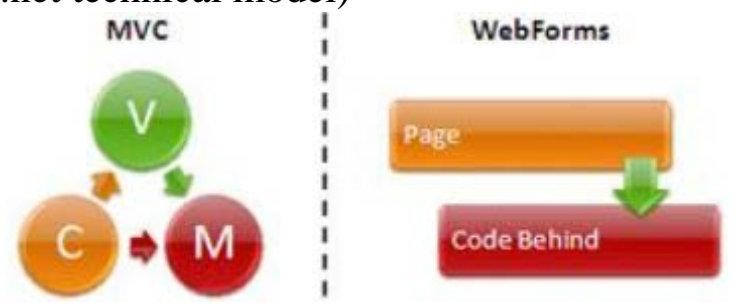

Figure 1 ASP net technical model

The application ASP.net technology of Web application three-layer design model is that its own advantages make up for the shortcomings of traditional technology.

ASP.Net as a Web application development technology, it is based on the Microsoft.net platform, on the common language running platform,. All the functions of the net Framework can be used. Using this technology to develop programs for exception control can play a supporting role, maintain type security, achieve inheritance and dynamic compilation.A variety of support surfaces can also be fully utilized in ASP.net programs to program control logic to a strong type of language. (Figure 2: Microsoft.net Platform)

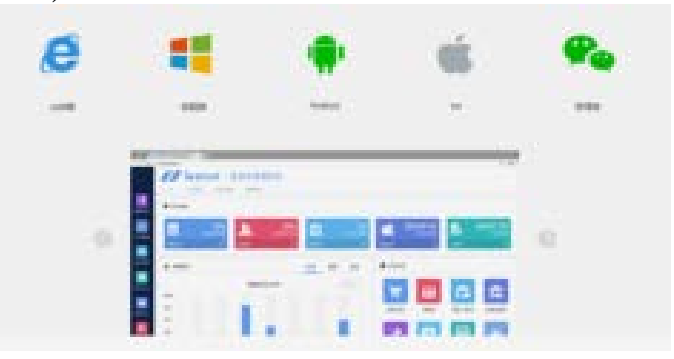

Figure 2 Microsoft.net platform

Web Form programming model is built based on ASP.Net technology in the process of 
developing program. Using this model, the interaction between client and server can be completed automatically from the underlying system, the state answering function can be realized, the automatic maintenance of various data information, and the function of server control, such as server control and man-machine interface control, etc. The form service control also supports data binding, and the powerful function can be developed by running simple code.

During ASP.net program, the page display section as well as the control logic section can be in. aspx documents and. Storage $\mathrm{c} x$ files, the two achieve separation, which makes the Web application run more stable, and later technical maintenance easier. Using ASP.net technology, we can use object-oriented design ideas and program editing methods to make the development of Web applications more efficient and better quality.Web the application has its own characteristics, using a reasonable design pattern and continuous optimization according to the actual needs, the net platform can be fully utilized to give full play to the advantages of ASP.net technology and the characteristics it has, and the well-run Web application can be developed and designed.

\section{Three-tier Design Model of Web Application Based on ASP.net Technology}

\subsection{Basic Introduction of the Layered Model}

A traditional Web application structure is usually two layers, which can be used if the database in the website environment is relatively small and the data is single. Applications are being scaled up, and if two-tier designs remain in place, the disadvantages are exposed, large enterprise wide area networks can not be used, and the application of the technology in the Internet can be difficult to maintain.At such a situation, it is necessary to innovate technology, better adapt to the network environment, ASP.net the application of technology to make up for the disadvantages of traditional technology, in fact, the use of three-layer design model, so that software program development is full of vitality.

Web itself is a distributed application, in the process of running requires the server side of the web server with the use of the client browser to play a good role, its various functions can be implemented, this is the B/S structure.Starting from the characteristics of the web and the ASP.net technical elements, the application is divided into three layers in the program development, and the design model is constructed, that is, the application structure is divided into user interface layer, business logic layer, data access layer, the three layers are closely linked, but also exist independently, each has its own unique functions and undertakes its own tasks.[1].

\subsection{Three-Tier Model Structure}

The structure of the three-layer design model is clear, simple and clear, and the operation is very convenient. The function of each layer is very clear, the task is also very clear, and there is a correspondence between the layers, which makes the program development work simplified.Each layer has the unique function, therefore has the independence, may play the certain promotion function to the program development efficiency enhancement. Each layer of the three-tier design model has good cohesion, which is very advantageous in object-oriented language programming. (Figure 3: web application three-tier design model)

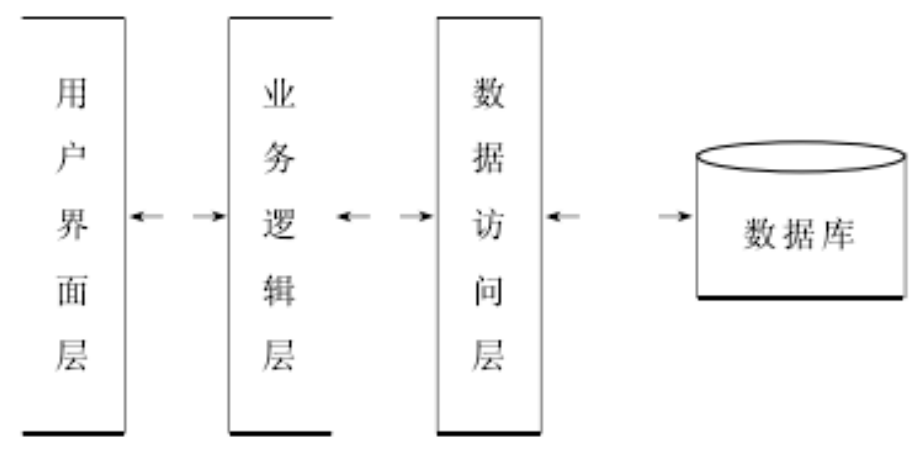

Figure 3 Web application three-tier design model 


\subsection{User Interface Layer}

There is a user interface display in the customer browser, this is the user interface layer, the business logic layer published a variety of data information are dynamically presented on this interface, and can be human-computer interaction, so that users and programs communicate. All the data information is displayed through HTML tags and cascading stylesheets. It can also input the data, check the data information, and transmit the data information to the business logic layer. The user interface layer and the business logic layer establish close relationship with each other, at the same time exert their respective functions and complete their respective tasks.

\subsection{Business Logic Layer}

In the three-tier model structure, the business logic layer is the most critical and in the middle position. This layer has a close relationship with the user interface layer, while interacting with the data access layer, which can not only provide function calls for the user interface layer, but also provide database access function for the data access layer.In the process of developing and designing the business logic layer, the whole project can be controlled based on several objects of engineering design. Specifically, the task of the business logic layer is to complete the dynamic data information of the mobile phone user interface layer, to call the data information of the data access layer, to save the data, or to transmit the data information to the user interface layer. The business logic layer is also tasked with collecting, saving and transmitting data information generated by the interaction between the processor and the other two layers.

The main function of the business logic layer is to realize the logic function at the field level and the logic function at the business level. To exchange information with the data access layer and make full use of the access function of the database on the data access layer, the function of the data access layer is called, the function of the user interface layer is provided and the business logic program is encapsulated. Such a design structure facilitates later technical maintenance and upgrading of procedures[2] .

One unit, for example, uses VS2010 and SQL Server2008 databases when creating a three-tier design model for a web application. The business logic layer is simple in design, plays the leading role, creates the Book BLL in the VS2010, then adds the BLL Get-Books method to the code, can effectively call the Books Table Adapters typed dataset class.

\subsection{Data Access Layer}

Among the three-tier design models of Web applications, the basic level is the data access layer, and all kinds of data access functions, such as page conversion function and data transmission function, are implemented in this layer, which not only operate quickly, but also are very convenient. In the process of running the application, if the data information needs to be changed due to the needs of the business logic layer, the data access layer can extract the relevant data from the database from the requirements, and then modify it from the requirements to ensure that the system is in a normal running state. Therefore, the data access layer is the lowest layer, which provides the guarantee for the normal operation of the three-layer design model. Of course, the resource consumption of this layer is also the largest, which needs to be continuously optimized to upgrade the database, so that its function is better and the function is more reliable. When designing this layer, if the database is SQL 2008,, the Table Adapter configuration can be fully utilized for the creation of the data access layer, directly connected with the database, exerting the function of language query and data information storage, and filling the results of the butterfly in the Data Table. When creating and editing data, it can be expanded in the form of typed data sets. During the development of the web application, the three-tier design model is popularized in an all-round way and plays a good role in the application field. The application structure is simplified, the relationship between the layers is clear, it is very beneficial for the technical maintenance and function upgrading of the system, and each layer is independent of each other, the efficiency of system development is improved, and the quality of system operation is guaranteed. 


\section{Conclusion}

Through the above research, we can make it clear that in the current social environment, the coverage of information network is becoming more and more extensive, and it is necessary to run three-tier design model in the development of website and software program editing.The web threelayer design model proposed in this paper is based on ASP.net technology and has been applied. It can be divided into three orderly layers from the specific working conditions, which is very beneficial to the operation and maintenance of the system.

\section{References}

[1] Yang, Xiao, Yin. ASP. NET MVC Architecture and Web Development Analysis. Automation and Instrumentation, no. 07, pp. 25-26, 2018.

[2] Ailing, Cheng. Yuanyu, Zhuang., Nanlin. Optimization of Control Data Paging Technology Based on ASP.NET. Journal of Chifeng College (Natural Science Edition), no. 05, pp. 98-99, 2019. 